\title{
Water adsorption and desorption pretreatment of surfaces increases the maximum amount of adsorbed water molecules by a multiple
}

\author{
Dietmar Neuhaus
}

Received: 22 January 2013/ Accepted: 28 February 2013/Published online: 16 March 2013

(c) The Author(s) 2013. This article is published with open access at Springerlink.com

\begin{abstract}
The amount of adsorbed water on surfaces in an atmosphere with $100 \%$ relative humidity can be increased by a multiple, if the surfaces are pretreated by cycles of adsorption and desorption of water. This was observed on surfaces of diamond, titanium dioxide and silicon dioxide at temperatures around $22{ }^{\circ} \mathrm{C}$. With a sufficient number of such cycles a faster and stronger adsorption of water molecules was obtained, if compared with untreated surfaces. This also means an increased energy transfer from the atmosphere to the surface. Due to the pretreatment the amount of adsorbed water was more than three times increased. The observed effect is explained by small amounts of specially arranged water molecules, which remain on the surface after the desorption process and which support the adsorption of water. The observed effect can be used to moisten surfaces of small particles very efficiently from the gas phase.
\end{abstract}

Keywords Water · Adsorption · Wetting ·

Activation cycles

\section{Introduction}

Water has great importance for the living conditions on earth and was for this reason investigated in many ways, as pure water, but also in combination with other substances. Especially about the interaction of water with solid surfaces an extensive literature exists (Thiel and Madey 1987, Henderson 2002), which also deals with the adsorption and

D. Neuhaus $(\bowtie)$

DLR, Institut für Materialphysik im Weltraum, Linder Höhe,

51147 Cologne, Germany

e-mail: dietmar.neuhaus@dlr.de desorption of water molecules from surfaces. Often the surfaces were prepared under ultrahigh vacuum conditions to obtain well defined surfaces. The adsorption of water at these low pressure must be performed at very low temperature to avoid immediate desorption of the water molecules. These kind of investigations are important, but it is questionable, whether results obtained in this low temperature range are sufficient to completely explain the adsorption and desorption of water from ambient air. Research results on the adsorption and desorption properties of water, with water vapor from atmospheres, which are known from the usual environments, are of special interest, because they are part of the everyday living conditions on earth. Every day on earth an enormous mass of water is transported between the atmosphere and solid surfaces, accompanied, due to the heat of adsorption/condensation and desorption/evaporation, with an huge energy transport. This paper deals with the adsorption of water on diamond, titanium dioxide and quartz surfaces, which were, at room temperature $\left(18-30{ }^{\circ} \mathrm{C}\right)$, exposed to an atmosphere with $100 \%$ relative humidity (RH). Although it will be shown that hundreds of monolayers of water were adsorbed on this surfaces it will be avoided to speak about condensation and evaporation, because the thickness of the water layer was limited, not typical for condensation.

\section{Experimental method}

Infrared spectroscopy with the attenuated total reflection (ATR) technique was used to measure the coverage of surfaces with water molecules (Fahrenfort 1961). ATR spectroscopy was selected because of its sensitivity to measure adsorbates on surfaces. The time behavior of adsorption was measured with a series of ATR spectra with 


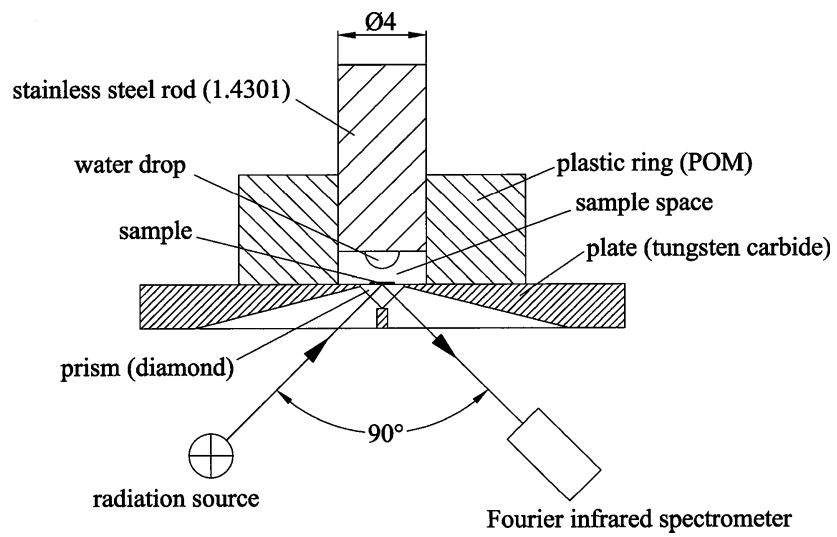

Fig. 1 Experimental set up to investigate the adsorption of water on surfaces of diamond, quartz and titanium dioxide with ATR infrared spectroscopy. The plastic ring and the stainless steel rod form a cap, which can be put on the plate to have the sample space. Due to the water drop there is $100 \% \mathrm{RH}$ in the sample space

$5 \mathrm{~s}$ time interval, which was in addition the measuring time for a single spectrum. A Fourier infrared spectrometer of the type Tensor27 of Bruker Optik GmbH, equipped with a device for ATR spectroscopy made by Specac, was used for these measurements. In Fig. 1 the experimental setup for the investigations is shown. The sample space in this setup is bounded by one of the side faces of a triangular right diamond prism with $4.37 \mathrm{~mm}^{2}$ surface area mounted in a tungsten carbide plate, the tungsten carbide plate itself, a ring made of POM plastic and a rod made of stainless steel (1.4301). The plastic ring with the steel rod forms a cap, which could be lifted from the tungsten carbide plate, to expose the diamond surface to the ambient air. Inside the sample space with $18.8 \mu \mathrm{L}$ volume an atmosphere with $100 \% \mathrm{RH}$ was obtained by placing a $2 \mu \mathrm{L}$ volume water drop (Merck Ultrapur) on the stainless steel rod. The poor wettability of the plastic was used to keep the water drop on the stainless steel rod. ATR spectroscopy was performed with the diamond prism. For this purpose the incident beam was totally reflected from the mentioned side face with $45^{\circ}$ incident angle. The total reflection causes an electromagnetic field which extends into the sample space and decreases exponentially with the distance to the surface of this side face. Substances, close to this side surface, which can absorb this radiation, cause an attenuation of the intensity of the reflected radiation, which can wavelength-dependent be measured with the Fourier spectrometer. The penetration depth is proportional to the wavelength $\lambda$ of the radiation used and is in the order of $\lambda /(2 \cdot \pi)$ (Born 1972).

It is known, that the infrared absorption spectrum of water in the wavelength range around $3,300 \mathrm{~cm}^{-1}$ shows a broad absorption band, which is related to $\mathrm{O}-\mathrm{H}$ stretching vibration modes of the water molecule. Details in this

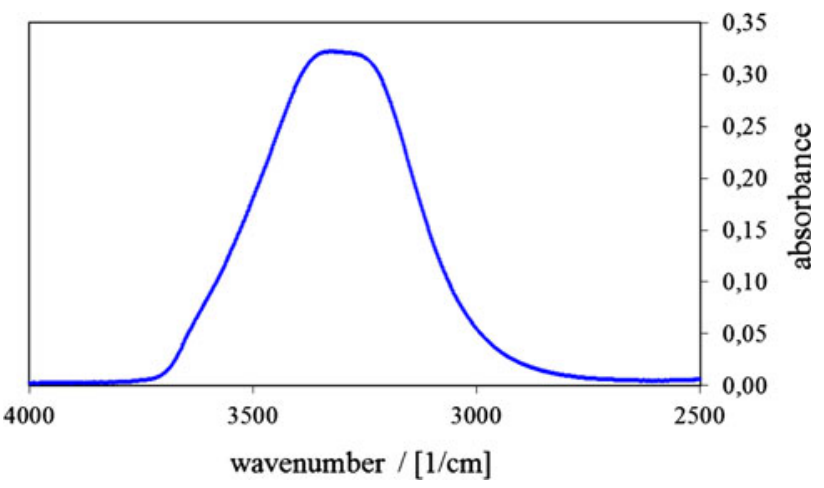

Fig. 2 ATR infrared spectrum of a water layer, approximately $2 \mathrm{~mm}$ thick, in the spectral range of the $\mathrm{O}-\mathrm{H}$ stretching vibration. Spectral resolution $2 \mathrm{~cm}^{-1}$

absorption band gives information about the arrangement of the water molecules.

Figure 2 shows the ATR infrared spectrum of an approximately $2 \mathrm{~mm}$ thick water layer, taken with $2 \mathrm{~cm}^{-1}$ spectral resolution at $26{ }^{\circ} \mathrm{C}$ temperature. The related reference spectrum was taken with the diamond surface exposed to the ambient air at $26^{\circ} \mathrm{C}$ and $66 \% \mathrm{RH}$ without any water coverage visible with the naked eye. Nevertheless, one must assume, that water was already adsorbed on the surface of the diamond, when measuring the reference spectrum. This assumption is obvious because of investigations of the adsorption of water on the surface $\mathrm{BaF}_{2}(111)$ made by Sadtchenko, Conrad and Ewing, who measured two monolayers of water on this surface at $25^{\circ} \mathrm{C}$ and $69.4 \%$ RH (Sadtchenko et al. 2002). In the wavelength range around $3,300 \mathrm{~cm}^{-1}$ the spectrum in Fig. 2 shows the mentioned strong absorption band of water, whose integrated absorbance in the wavelength range from 3,000 to $3,600 \mathrm{~cm}^{-1}$ will be taken in the following as a measure of the thickness of the adsorbed water layer. A typical adsorption experiment lasts $20 \mathrm{~min}$. During this short period of time it was ensured that temperature and $\mathrm{RH}$ in the laboratory did not change, to keep the experimental setup as stable as possible. Single samples were investigated over months. In this long period of time temperature and RH in the laboratory changes. So adsorption experiments were performed at different temperatures $\left(18-30{ }^{\circ} \mathrm{C}\right)$ and different $\mathrm{RH}$ in the ambient air (40-70\%). The observed effect reported here was not noticeably affected by this changes in temperature and $\mathrm{RH}$ in the ambient air.

\section{Results and discussion}

The description of adsorption experiments presented here starts with experiments on a diamond surface. In these experiments a water drop with $2 \mu \mathrm{L}$ volume was deposited 
on the front surface of the stainless steel rod of the cap, which was put on the tungsten carbide plate with the diamond, as shown in Fig. 1. Due to the water drop a sample space with $100 \% \mathrm{RH}$ was obtained (temperature $23.7^{\circ} \mathrm{C}$, $\mathrm{RH}$ in the ambient atmosphere $47 \%$ ). The surface of the diamond was exposed to the $100 \% \mathrm{RH}$ atmosphere for $2,000 \mathrm{~s}$. During this time period a maximum relative change of the integrated absorbance of only $<0.3 \mathrm{~cm}^{-1}$ was measured, which means, that only a small amount of water was adsorbed, during this time.

The adsorption of water on the diamond surface developed completely different, if, periodically, the cap with the water drop was first put on the tungsten carbide plate with the diamond for a certain time, then removed for a short period of time, put on again and so on. The observed behavior of water adsorption with this kind of prior treatment is shown with two adsorption experiments in Fig. 3 (blue and red curve). In this figure the blue curve describes the adsorption of water on a diamond surface as function of the time at $22.6{ }^{\circ} \mathrm{C}$ room temperature and with $56 \% \mathrm{RH}$ in the ambient air. The absorbance and therefore the coverage with water was measured in $5 \mathrm{~s}$ time intervals. The $5 \mathrm{~s}$ time resolution was used in nearly all adsorption experiments reported here. In the experiment the sequence of ATR spectra was started without the cap on the plate, then, $25 \mathrm{~s}$ later, the cap with the water drop was put on the plate and was, after every $115,205,295,385$ and $475 \mathrm{~s}$, removed for $5 \mathrm{~s}$ and replaced again. Immediately after removing the cap, the water, adsorbed under $100 \% \mathrm{RH}$, desorbed. In this case in an ambient atmosphere with $56 \% \mathrm{RH}$. The process of adsorption at $100 \% \mathrm{RH}$ followed by desorption in an ambient atmosphere with $<100 \% \mathrm{RH}$ is called an activation cycle, the selected period of time for adsorption, the adsorption time, may be selected different in length. With 5 activation cycles significantly more water was adsorbed in an atmosphere with $100 \% \mathrm{RH}$ than without activation. However, 1,100 s after experiment start the water coverage on the diamond surface starts to decrease continuously, although the cap with the water drop was still on the plate. The adsorption experiment without activation is shown in the green curve in Fig. $3\left(23.7{ }^{\circ} \mathrm{C}\right.$ room temperature, $47 \%$ $\mathrm{RH}$ in the ambient air). In another experiment, in Fig. 3 represented by the red curve, 13 activation cycle, each with $45 \mathrm{~s}$ adsorption time, were performed $\left(22.5^{\circ} \mathrm{C}\right.$ room temperature, $57 \% \mathrm{RH}$ in the ambient air). In the subsequent adsorption of water, in an atmosphere with $100 \%$ $\mathrm{RH}$, measured with series of ATR spectra, a steady increase of the water coverage was observed, until, after approximately $1,150 \mathrm{~s}$, the maximum was reached, which did not change till the end of the experiment. The thickness of this water coverage can be estimated by comparing these ATR spectra with the mentioned ATR spectra measured with the $2 \mathrm{~mm}$ thick water layer on the diamond surface.

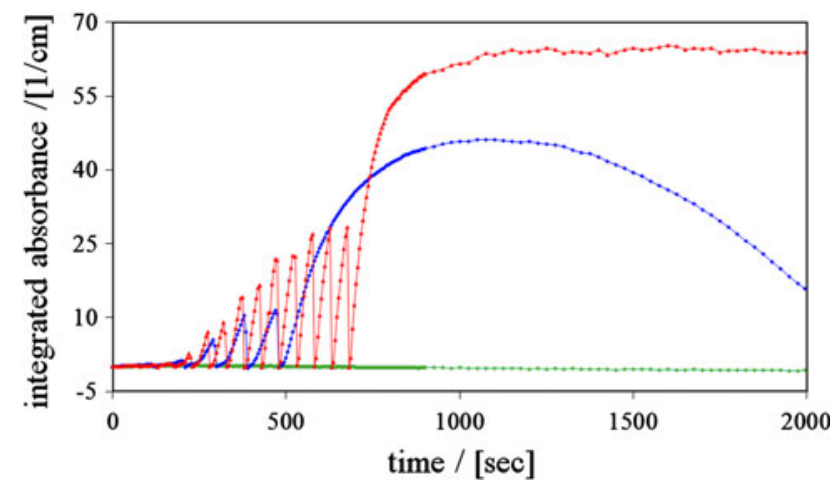

Fig. 3 Adsorption of water on a diamond surface as a function of time. The adsorbed quantity of water was measured by the integrated absorbance in the spectral range from 3,000 to $3,600 \mathrm{~cm}^{-1}$. With activation blue and red curve, without activation green curve. The red curve belongs to the adsorption of water with a wetting disaster

The exponential decline of the magnitude of the electrical field strength $\mathrm{E}$ in the sample space with the distance $z$ to the diamond surface is related to the formula $E=E_{0}$. $\exp \left(\frac{-2 \cdot \pi \cdot z}{\lambda} \cdot \sqrt{n_{1}^{2} \cdot \sin \theta^{2}-n_{2}^{2}}\right)\left(E_{0}\right.$ magnitude of the electrical field strength at the interface between diamond and water, $n_{1}=2.38$ refractive index of diamond, $n_{2}=1.42$ refractive index of water, $\theta=45$ angle of incidence, $\lambda$ wavelength) (Born 1972). In $2 \mu \mathrm{m}$ distance from the diamond/water interface the electrical field strength is already lower than $3 \%$ of the electrical field strength at the interface, calculated for wavenumber $3,300 \mathrm{~cm}^{-1}$. It follows, that the ATR spectra of the water layer in Fig. 2 will not be distinguishable from an ATR spectrum of an even thicker water layer. With this data, a maximum thickness of the layer of water, adsorbed after 13 activation cycles, can be calculated to approximately $0.18 \mu \mathrm{m}$. Thickness of a water monolayer $\approx 25 \mathrm{~nm}$ (Miranda et al. 1998).

The adsorption of water on quartz and titanium dioxide surfaces was measured with fine quartz flour and fine titanium dioxide powder (anatase). Quartz flour SILMIKRON VP795-10/1 provided by Quarzwerke GmbH was used. The particle size distribution of the quartz flour was described with the cumulative distribution function with $d_{90 \%}=1 \mu \mathrm{m}, d_{50 \%}=0.5 \mu \mathrm{m}$ and $d_{10 \%}=0.2 \mu \mathrm{m}$ taken from the data sheet. With respect to the data sheet of the titanium dioxide powder KRONOS 1001, produced by Kronos Titan $\mathrm{GmbH}$, the particle sizes were comparable to the sizes of the quartz flour particles, but, the distribution of the titanium dioxide particle sizes was broader with $d_{16 \%}=0.21$ $\mu \mathrm{m}, d_{50 \%}=0.51 \mu \mathrm{m}$ and $d_{84 \%}=1.06 \mu \mathrm{m}$ of the cumulative distribution function.

For the adsorption experiments suspensions of quartz flour and titanium dioxide powder with water were prepared. A drop of a suspension with $2 \mu \mathrm{L}$ volume was placed on the ATR diamond surface and dried in ambient 


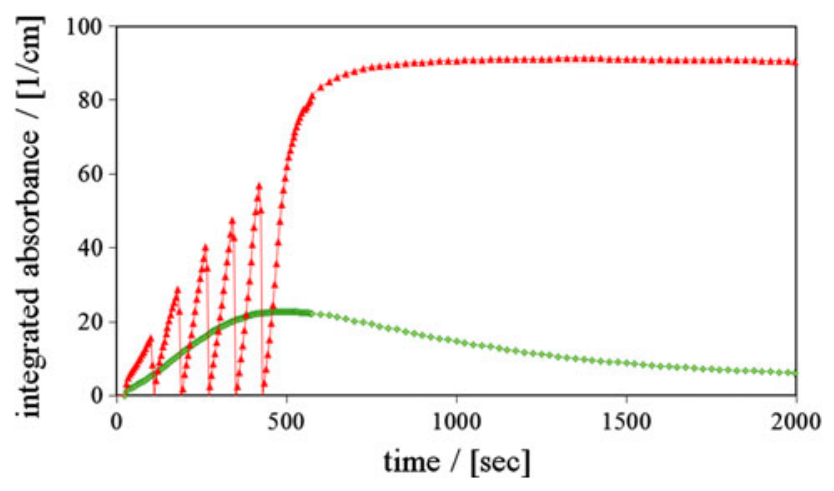

Fig. 4 Adsorption of water on the sample quartz flour (SILMIKRON VP795-10/1) on diamond. The quantity of the adsorbed water is measured by the integrated absorbance (wavenumber range $3,000-3,600 \mathrm{~cm}^{-1}$ ) as a function of time. Without activation green curve, with activation red curve. The activation was sufficient for a wetting disaster

air with temperatures around $25^{\circ} \mathrm{C}$ to form a sample for adsorption experiments. After this procedure the diamond surface was covered with a thin layer of quartz or titanium dioxide particles, with $27.3 \mu \mathrm{g}$ quartz particles or $22.8 \mu \mathrm{g}$ titanium dioxide particles distributed on the $4.37 \mathrm{~mm}^{2}$ area ATR diamond surface. With such samples water adsorbs on the diamond surface and the surface of the particles as well, and the ATR spectra measure the water coverage on both surfaces. It can be expected, that the absorbance of the diamond surface with particles is higher, due to the increased surface area of the diamond surface with the particles, if compared with a clean diamond surface. The area fraction of the diamond as part of the total surface area accessible to ATR spectroscopy can be estimated to be lower than $36 \%$, if it is assumed, that the particles on the surface are closed packed, only the first layer of the particles are taken into account and only the undersides of the particles are considered.

Figure 4 shows the results of two experiments of adsorption of water on the quartz sample mentioned above. The green and the red curve describes the adsorption of water as function of the time in an atmosphere with $100 \%$ RH. Green curve $19.6{ }^{\circ} \mathrm{C}$ room temperature and no activation of the surface, red curve $19.5{ }^{\circ} \mathrm{C}$ room temperature and with activation of the surface. Five activation cycles were performed, with $75 \mathrm{~s}$ adsorption time $(\mathrm{RH}$ in the ambient air $41 \%$ ). Approximately $700 \mathrm{~s}$ after experiment start a stable water coverage was observed. Without activation, in an atmosphere with $100 \% \mathrm{RH}$, the water coverage grows up in the beginning but then starts to decline approximately $480 \mathrm{~s}$ after the cap was put on the plate.

Figure 5 shows the results of adsorption experiments of water on the sample: titanium dioxide powder (Kronos 1001) on diamond. The preparation of the sample was described above. Similar to the observations with the

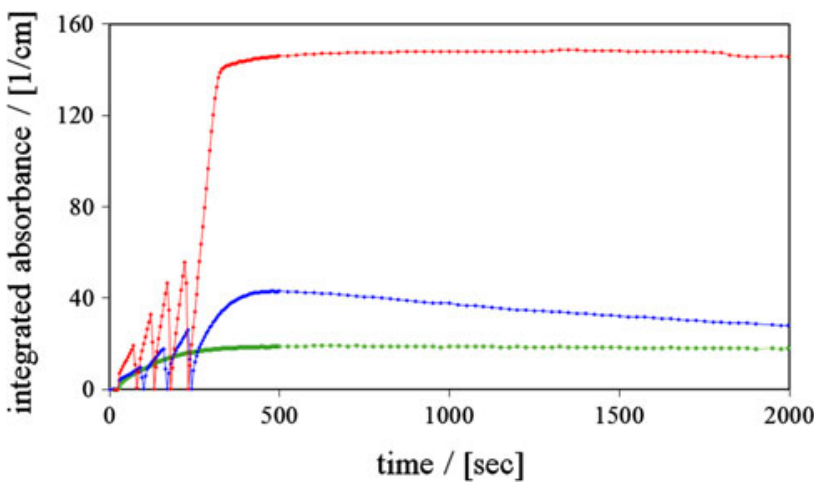

Fig. 5 Adsorption of water on the sample titanium dioxide powder (Kronos 1001) on diamond. The quantity of the adsorbed water is measured by integrated absorbance as a function of time (wavenumber range 3,000-3,600 $\mathrm{cm}^{-1}$ ). Without activation green curve, with activation blue and red curve. The red curve describes an adsorption experiment with a sufficient activation of the surface to have a wetting disaster

sample quartz flour on diamond also with this sample a stable water coverage was built up in an atmosphere with $100 \%$ RH only after a sufficient activation of the surface of the sample. With three activation cycles, each with $65 \mathrm{~s}$ adsorption time, a stable water coverage could not be achieved (blue curve $28.8{ }^{\circ} \mathrm{C}$ room temperature, $54 \% \mathrm{RH}$ in the ambient atmosphere), in an other experiment four activation cycles with $45 \mathrm{~s}$ adsorption time where sufficient to obtain a stable water coverage in $100 \% \mathrm{RH}$ (red curve, room temperature $21.7{ }^{\circ} \mathrm{C}$, ambient atmosphere $49 \% \mathrm{RH}$ ). Again, without activation, less water is adsorbed on this sample. This shows the green curve in Fig. 5 measured at $22{ }^{\circ} \mathrm{C}$ room temperature (62\% $\mathrm{RH}$ in the ambient air).

The described experiments show, that the amount of water adsorbed on surfaces of diamond, quartz and titanium dioxide in an atmosphere with $100 \% \mathrm{RH}$ can be increased by prior activation cycles. With a prior, sufficient activation of the mentioned surfaces, water molecules from an atmosphere with $100 \% \mathrm{RH}$ adsorb on this surfaces and form a strong and stable water coverage, which is called in the following a wetting disaster. Obviously the activation of the surface is related to the desorption of adsorbed water molecules. The more water desorbs in an activation cycle, the stronger becomes the activation. This follows from the increase of the maximum of the integrated absorbance during a single activation cycle from one activation cycle to the following one as shown in the Figs. 3, 4 and 5. The increase of the maximum of the integrated absorbance and therefore the increase of the activation becomes less with increasing number of activation cycles.

The activation level of the investigated surfaces for the adsorption of water was not permanent, after the activation process was finished the activation level decreases with time. This results from adsorption experiments with the 


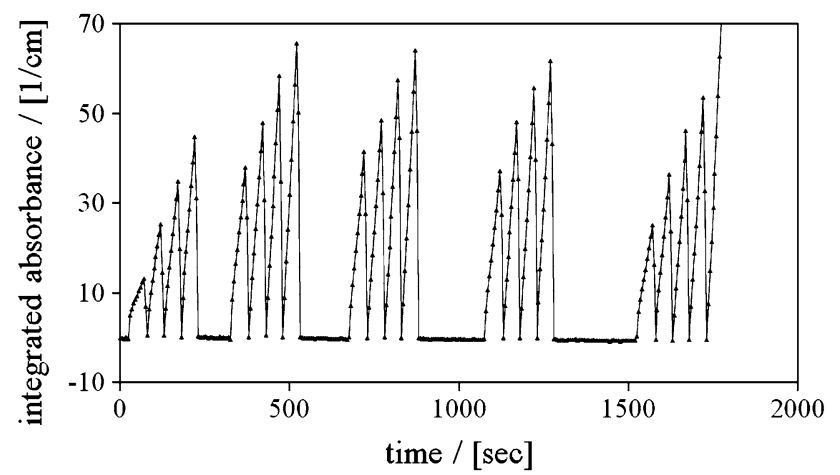

Fig. 6 Activation of the surface of the sample titanium dioxide powder (Kronos 1001) on diamond by adsorption and desorption cycles of water. The measure of the water coverage during adsorption and desorption as a function of time is the integrated absorbance (wavenumber range $3,000-3,600 \mathrm{~cm}^{-1}$ ). The activation cycles are organized in five groups with 4 single activation cycles. The time between the groups of activation cycles, with no cap on the plate, was $100,150,200$ and $250 \mathrm{~s}$

mentioned sample surfaces, when the surfaces were treated by groups of activation cycles and otherwise exposed to an atmosphere with less than $60 \% \mathrm{RH}$. The time intervals between the groups were selected longer than the respective adsorption time in a single activation cycle. Figure 6 shows the result of such an experiment with the sample titanium dioxide powder on diamond with $24{ }^{\circ} \mathrm{C}$ room temperature and $43 \% \mathrm{RH}$ in the ambient atmosphere. Between the five groups, each with 4 activation cycles and always $50 \mathrm{~s}$ adsorption time, the cap with the water drop was lifted from the plate for 100, 150, 200 and $250 \mathrm{~s}$ time. The maximum of the integrated absorbance of the first activation cycle in the second group of activation cycles is smaller than the maximum integrated absorbance of the last activation cycle of the first group of activation cycles. The same applies to the relation of the third group of activation cycles to the second group and the fourth group to the third and the fifth group to the fourth group. This makes clear, that the activation of the surface of the sample titanium dioxide on diamond has been reduced in the time between the groups of activation cycles.

What is the reason for the observed activation of the surfaces for the adsorption of water molecules?

Diamond and titanium dioxide are insoluble in water (Falbe and Regitz 1999, p. 933 and 4564), it is therefore not expected, that the activation cycles will change the structure of the surfaces of these substrates. In contrast, quartz has $10.8 \mathrm{ppm}$ solubility in water at $25^{\circ} \mathrm{C}$ temperature (Rimstidt 1997), so that it can not be excluded, that the activation cycles change the structure of the quartz surface. But, the similarity of the observed activated adsorption on the mentioned surfaces (Figs. 3, 4, 5) indicate, that the state of an activated surface is the same in all considered cases. Because of the insolubility of diamond and titanium dioxide in water, this can not be a change of the structure of the substrate surface itself.

The activated surface could be a surface with a small amount of adsorbed water molecules, probably specially arranged, which, after an activation cycle, remain on the surface. Such an interpretation of the experimental results seems reasonable, if it is true, that the surface of the substrate will not be changed by the activation cycles.

ATR infrared spectroscopy was used to find evidence for these assumed water molecules in the spectral range of the $\mathrm{O}-\mathrm{H}$ stretching vibration modes of water. A large surface area would be advantageous for this kind of investigation, so the sample titanium dioxide on diamond was selected for the experiments. Looking for these assumed water molecules only a short period of time, some seconds after an activation cycle, would probably be available to measure the infrared spectrum in a single adsorption experiment. This suggests only a poor signal to noise ratio of such a spectrum. To improve this, a number of 37 similar adsorption experiments were performed and the mean of the measured spectra calculated. Each of these adsorption experiments were characterized by 12 activation cycles with $40 \mathrm{~s}$ adsorption time and the occurrence of a subsequent wetting disaster. In these experiments the time between the activation cycles and the time between the last activation cycle and the cap again on the plate was increased from the normally used 5 to $10 \mathrm{~s}$. Two ATR spectra, each requires $5 \mathrm{~s}$ measuring time, could be measured in the time period without the cap on the plate. In Fig. 7 three ATR spectra Sp0, Sp5 and Sp10 are shown, obtained by averaging of ATR spectra from the 37 mentioned adsorption experiments. Three groups of ATR spectra were averaged, first the spectra measured immediately after the last activation cycle ( $\mathrm{Sp} 0)$ and two others measured $5 \mathrm{~s}(\mathrm{Sp} 5)$ and $10 \mathrm{~s} \mathrm{(Sp10)}$ after the last activation cycle (temperature range $21.2-27.5^{\circ} \mathrm{C}, \mathrm{RH}$ in the ambient atmosphere 40-64\%). The spectra Sp0 and Sp5 were measured without the cap on the plate, both spectra show only a small and very noisy signal of the absorbance in the spectral region of the $\mathrm{O}-\mathrm{H}$ stretch vibration of water. In both spectra the corresponding moving average of the order 40 was added. The spectrum Sp10 is related to spectra measured immediately after the cap with the water drop was put on the plate, it shows, compared with the spectra $\mathrm{Sp} 0$ and $\mathrm{Sp} 5$, an approximately 50-fold higher absorbance. Comparing the maximum of the spectra $\mathrm{Sp} 0, \mathrm{Sp} 5$ and $\mathrm{Sp} 10$ we have a shift of the maximum of $\mathrm{Sp} 0$ at $3,290 \mathrm{~cm}^{-1}$ and of $\mathrm{Sp} 5$ at $3,359 \mathrm{~cm}^{-1}$ in the direction of smaller wave numbers if compared with the maximum of Sp10 at $3,391 \mathrm{~cm}^{-1}$. In a paper by Smith et al. (2002) a shift of the maximum in the spectral range of the $\mathrm{O}-\mathrm{H}$ stretching vibration modes was correlated with changes of the 


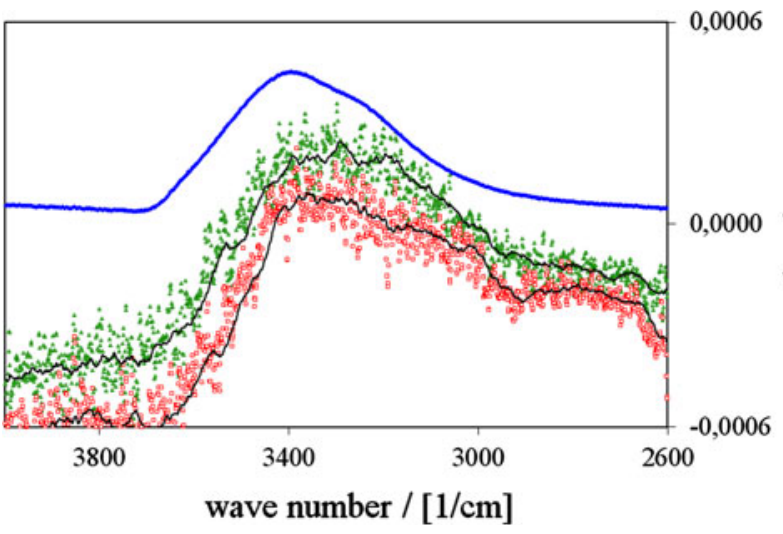

Fig. 7 ATR infrared spectra $\mathrm{Sp} 0$ (red squares), Sp5 (green triangles) and Sp10 (blue diamonds) of the sample titanium dioxide on diamond. The spectra Sp0 and Sp5 are ATR infrared spectra of an activated surface. The spectrum Sp10 was measured immediately after the cap with the water drop was put on the plate. All spectra are mean values taken from a series of adsorption experiments. The black curves are moving averages of the mean values of the spectra $\mathrm{Sp} 0$ and Sp5. The scale of the integrated absorbance of spectrum Sp10 was reduced by the factor 50

arrangements of the water molecules among themselves. Smith et al. have investigated water adsorbate on a platinum $\mathrm{Pt}(111)$ surface at low temperatures (80-146 K), in this investigation also infrared reflection spectroscopy was used, and it was observed, that the shift of the maximum in the direction of smaller wave numbers means a transition from an amorphous to a crystalline structure of the adsorbed water molecules. The amorphous water layer shows in the reflection infrared spectrum, in the spectral range of the $\mathrm{O}-\mathrm{H}$ stretching vibrations, a maximum at $3,410 \mathrm{~cm}^{-1}$; the crystalline water layer showed a maximum shifted to lower wave numbers at $3,260 \mathrm{~cm}^{-1}$. This interpretation of the infrared reflection spectra of adsorbed water with respect to the arrangement of the water molecules will be applied to the interpretation of the ATR spectra of adsorbed water, adsorbed on the surfaces of the mentioned three samples, presented here. The spectra $\mathrm{Sp} 0$ and $\mathrm{Sp} 5$ are ATR spectra from activated surfaces. If it is true, that residual water molecules on the surfaces are responsible for the activation, then these molecules are arranged in a partly crystalline fashion. The adsorption of water after the activation initially increases the portion of the amorphous phase in the adsorbate, which, with increasing water coverage, becomes more and more crystalline. This transition from amorphous to crystalline was not only observed on the sample titanium dioxide on diamond, but also, as shown in Fig. 8, on the two other samples. Although in each case at maximum coverage the wetting disaster occurred and temperature and $\mathrm{RH}$ in the ambient air was comparable (diamond $22.5^{\circ} \mathrm{C}$, $57 \% \mathrm{RH}$, quartz flour on diamond $19.5^{\circ} \mathrm{C}, 41 \% \mathrm{RH}$, titanium dioxide on diamond $\left.21.7^{\circ} \mathrm{C}, 49 \% \mathrm{RH}\right)$, the

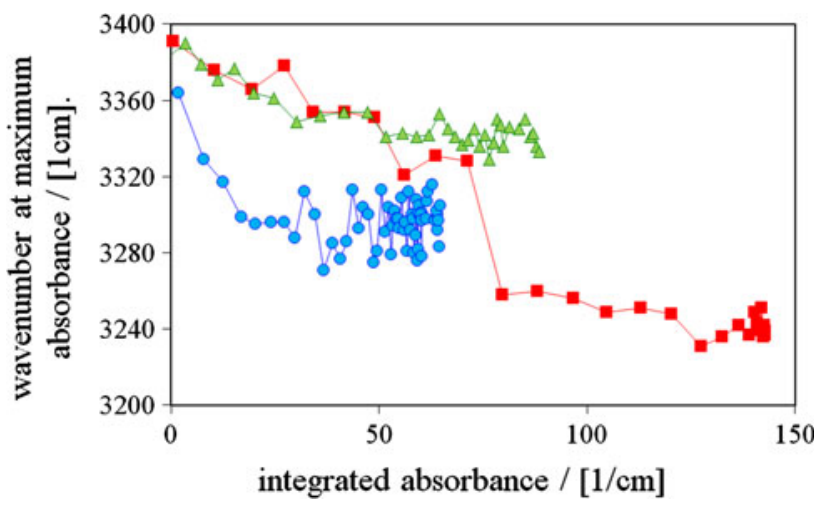

Fig. 8 Wavenumber at the maximum of the absorbance in the wavenumber range of the $\mathrm{O}-\mathrm{H}$ stretching vibration as function of the integrated absorbance (wavenumber range 3,000-3,600 $\mathrm{cm}^{-1}$ ), for the samples diamond (blue dots), titanium dioxide powder on diamond (red squares) and quartz flour on diamond (green triangles)

observed degree of crystallization at this coverage was different.

The series of ATR infrared spectra, measured during the adsorption of water on the samples, were used to look for differences in the shape of the spectra in the spectral range of the $\mathrm{O}-\mathrm{H}$ stretching vibration modes, depending on whether activation cycles were previously performed or not. For this investigation the comparison of spectra with the same integrated absorbance is of particular interest because in this case the amount of adsorbed water is the same but the structure of the arrangement of the water molecules might not be. Figure 4 shows, as already mentioned above, the adsorption of water on the quartz sample. The green curve is related to an adsorption experiment without and the red curve with activation of the surface and the two curves intersect at several time points. At around $458 \mathrm{~s}$ on the time line an intersection of the two curves can be observed. ATR spectra measured at $460 \mathrm{~s}$ are closest to this intersection point and have the integrated absorbance $22.69 \mathrm{~cm}^{-1}$ green curve and $24.65 \mathrm{~cm}^{-1}$ red curve. The adsorption experiment related to the red curve shows a wetting disaster. These two spectra were compared and the results are shown in the left part (a) of Fig. 9. In this figure the absorbance scale of one of the two spectra were lineraly addjusted to have the same absorbance at $3,800 \mathrm{~cm}^{-1}$ and the same area under the curves between 3,000 and $3,600 \mathrm{~cm}^{-1}$ for both spectra. Indicated by arrows, slight differences in the shape of the $\mathrm{O}-\mathrm{H}$ streching vibration band are visible. In the red curve the high wavenumber area of the band is slightly decreased and the low wavenumber area slightly increased, if compared with the spectrum without previous activation of the surface (green curve). This effect is more intense in case of the titanium dioxide sample. In Fig. 5 the adsorption of water on the titanium dioxide sample with and without activation of the 
Fig. 9 ATR infrared spectra with (red curves) and without (green curves) previous activation of the surface. Arrows indicate changes in the spectra due to the activation. The left part of the figure (a), is related to the quartz sample and the right part (b), to the titanium dioxide sample

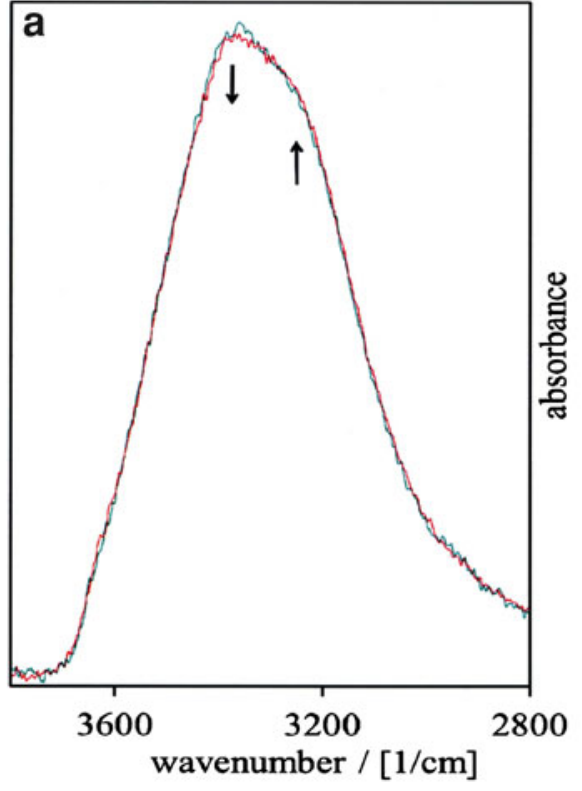

surface is shown. Here again the intersection of the green and the red curve was selected to compare another pair of the spectra measured at $240 \mathrm{~s}$. In this case the integrated absorbance was $16.36 \mathrm{~cm}^{-1}$ (green curve, without activation) and $19.52 \mathrm{~cm}^{-1}$ (red curve, with activation and wetting disaster). The result is shown in the right part (b) of Fig. 9, again the absorbance scale of one of the two spectra were linearly adjusted to have the same absorbance at $3,800 \mathrm{~cm}^{-1}$ and the same area under the curves between 3,000 and $3,600 \mathrm{~cm}^{-1}$ for both spectra.

If the mentioned interpretation of Smith et al. (2002) related to the shape of the $\mathrm{O}-\mathrm{H}$ stretching vibration band is true, this would mean, that with the same amount of adsorbed water the arrangement of the water molecules on the previously activated surface is more crystalline than on the not activated surface.

In a paper by Miura et al. (1999) the adsorption of water on lithium fluoride, calcium fluoride and barium fluoride single crystal surfaces was investigated with an atomic force microscope. These surfaces were exposed to an atmosphere with $30 \% \mathrm{RH}$ at room temperature for several minutes. In these experiments it was observed, that water molecules on these surfaces form islands, which means, that water layers are formed by island growth.

In the following it is tried to describe the observed activated adsorption of water with an island growth mechanism. In the model only the activated adsorption of water in an atmosphere with $100 \% \mathrm{RH}$ will be considered. The activation of the surface is assumed to be noticeable in the adsorption probability of water molecules, which hit the surface. It is further assumed, that the adsorbed water molecules initially form a surface gas which condensates on nucleation sites to water islands. The direct exchange of water molecules from the islands and water molecules from the atmosphere are expected to be balanced, because of the $100 \% \mathrm{RH}$ in the atmosphere. With these assumptions the water islands grow or shrink by the exchange of water molecules from the surface gas over the edges of the water islands. This exchange process is considered to be rate determining for the activated adsorption, because the balance between the water concentration in the surface gas and the atmosphere will be obtained within microseconds, due to the high vapor pressure of water at room temperature $\left(23.4 \mathrm{mbar}\right.$ at $\left.20{ }^{\circ} \mathrm{C}\right)$. It is also assumed, that the density of the surface gas is related to the activation of the surface, it is taken as a measure of the degree of activation of the surface. A description of time dependence of the density of the surface gas, after the activation procedure and starting at that time when the cap is back on the plate, was attempted by Eq. (1).

$n=n_{\infty}+n_{0} \cdot e^{-\frac{t}{\tau}}$

where $n$ is the density of the surface gas, $t$ time, $\tau$ the time constant, $n_{0}$ the prefactor, $n_{\infty}$ is the base density of the surface gas.

In this approach the density of the surface gas is described by time dependent and not time dependent summands. The time-independent summand $n_{\infty}$ was chosen to explain the observed wetting disaster in the experiments; the time-dependent summand takes into account the decline of the degree of activation with time, also observed in the experiments. The attenuation rate is hereby assumed to be proportional to the time-dependent summand, therefore the exponential time dependence of the attenuation was selected. The time-independent summand in Eq. (1) is assumed to be time-independent for at least the time period 
of the described adsorption experiments, which lasted approximately $30 \mathrm{~min}$. For time periods of several hours this assumption is no longer true, this was shown with adsorption experiments, performed in distances of hours after activation. The volume change rate of the water islands is assumed to be proportional to the length of the edge of the water islands $L$ and the difference between the density of the surface gas $n$ and the equilibrium density of the surface gas $n_{g}$. The equilibrium density $n_{g}$ is defined as the density of the surface gas where no change of the volume of the islands occurs.

$\frac{\partial V}{\partial t}=\chi \cdot L \cdot\left(n-n_{g}\right)$

where $V$ is the volume of the water islands, $\chi$ is the proportionality factor

For the subsequent calculations a water island is in a first approximation described by a cuboid with a quadratic base area. The length of the edge of the square base of the cuboid is equal $s$, the height of the cuboid $h$ is set equal to the height of the water island. If $N$ is the number of the nucleation sites on the surface and it is assumed that all water islands are equal, the volume and the edge length of the water islands can be calculated to be:

$V=N \cdot s^{2} \cdot h$

$L=N \cdot 4 \cdot s$

Using Eqs. (1), (3) and (4) with Eq. (2) yields the differential Eq. (5), when it is additionally assumed, that after the activation, the number of the nucleation sites and the height of the water islands on the surface will not change.

$\frac{\partial s(t)}{\partial t}=2 \cdot \frac{\chi}{h} \cdot\left(n_{\infty}-n_{g}+n_{0} \cdot e^{-\frac{t}{\tau}}\right)$

With the boundary condition $s(0)=0$ we have the solution of the differential equation:

$s(t)=2 \cdot \frac{\chi}{h} \cdot\left(n_{0} \cdot \tau \cdot\left(1-e^{-\frac{t}{\tau}}\right)+\left(n_{\infty}-n_{g}\right) \cdot t\right)$

It is assumed, that the measured absorbance $A$ of the adsorbed water molecules is mainly the absorbance of the water islands, because of the higher density of the water molecules in the islands, if compared with the surface gas. The absorbance is therefore assumed to be proportional to the volume of the water islands (proportionality factor $\alpha$ ). This means:

$A(t)=\alpha \cdot N \cdot \frac{4 \cdot \chi^{2}}{h} \cdot\left[n_{0} \cdot \tau \cdot\left(1-e^{-\frac{t}{\tau}}\right)+\left(n_{\infty}-n_{g}\right) \cdot t\right]^{2}$

Within this model the wetting disaster requires the condition $n_{\infty}=n_{g}$. In case of $n_{\infty}<n_{g}$, in the behavior of adsorption, first an increase of the absorbance with time is

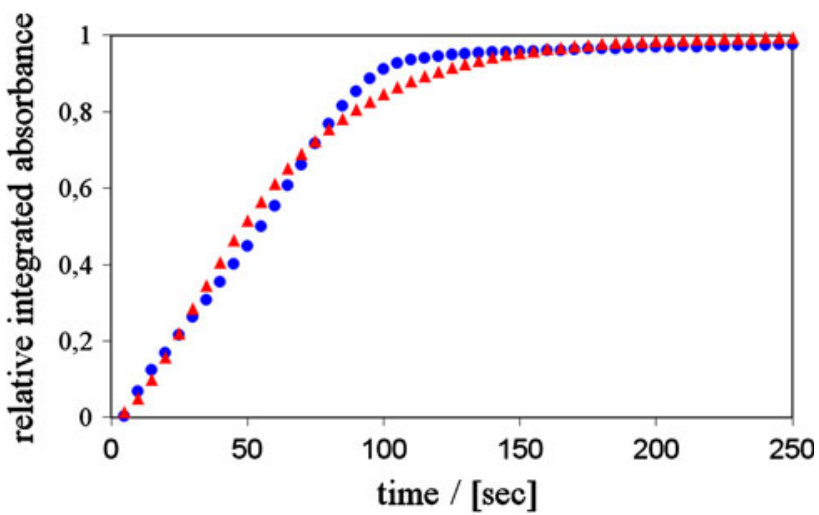

Fig. 10 Comparison of the measured (blue dots) with the calculated (red triangles) adsorption of water on the sample titanium dioxide powder on diamond as function of time in an atmosphere with $100 \%$ RH. The sample was sufficient activated to have a wetting disaster. The measured water coverage is shown as relative integrated absorbance (wavenumber range $3,000-3,600 \mathrm{~cm}^{-1}$ ), related to the maximum of the measured integrated absorbance. In the calculation the time constant $\tau=39.4 \mathrm{~s}$ was used

observed, but then it decreases, which is typical for an adsorption experiment with an activation of the surface not sufficient to have a wetting disaster. Adsorption experiments with wetting disaster were analyzed with the help of Eq. (7). Using the least square method the model parameter were fitted to the experimental data of the adsorption experiments with the sample titanium dioxide powder on diamond. Within the model a time constant $\tau=39.4 \pm 7 \mathrm{~s}$ was obtained as the mean value of 12 analyzed experiments, measured at different temperatures in a temperature range from 19 to $29{ }^{\circ} \mathrm{C}$ and a $\mathrm{RH}$ in the ambient air from 48 to $58 \%$. A clear temperature dependence of $\tau$ in this temperature range was not observed. In Fig. 10 one of these measured curves $\left(19{ }^{\circ} \mathrm{C}\right.$, ambient air $\left.54 \% \mathrm{RH}\right)$ in combination with the curve calculated according to the model with $\tau=39.4 \mathrm{~s}$ is shown.

\section{Conclusion}

In this work it was shown that the amount of adsorbed water on the surfaces of diamond, quartz and titanium dioxide depends on prior adsorption and desorption cycles of water on these surfaces. The adsorption and desorption cycles of water molecules, the activation cycles, activates the surface so that water adsorbs faster and in a much higher quantity in an atmosphere with $100 \% \mathrm{RH}$, if compared with the adsorption of water on a not activated surface. The activation is explained by small amounts of water molecules remaining on the surface after nearly all adsorbed water molecules have been desorbed. This residual water molecules have partly a crystalline structure. 
The surfaces are efficiently activated by a series of activation cycles, performed one after the other. With a prior sufficient number of activation cycles the investigated surfaces show a wetting disaster, a strong adsorption of water, when exposed to an atmosphere with $100 \% \mathrm{RH}$.

It is assumed, that this effect is not restricted to the surface of the samples diamond, quartz flour and titanium dioxide powder, but can also be observed on other surfaces. If this assumption is true, then the observed effect is a special property of water.

Quartz is a widespread mineral in the crust of the earth, the activated adsorption of water affects therefore also the important exchange of water molecules between the soil of the earth and the water in the atmosphere. An activation would increase the amount of exchanged water and also the amount of exchanged energy. Possibly, this can be relevant for the climate on earth.

In technical applications the activated adsorption effect can be used to moisten surfaces with water from an atmosphere much stronger and more rapidly. This can accelerate production processes in the chemical and the food industry, when, for example, granular media must be moistened by water from the gas phase.

Open Access This article is distributed under the terms of the Creative Commons Attribution License which permits any use, distribution, and reproduction in any medium, provided the original author(s) and the source are credited.

\section{References}

Born, M.: Optik, p. 41. Springer Verlag, Berlin, Heidelberg, New York (1972)

Fahrenfort, J.: Attenuated total reflection: a new principle for the production of useful infra-red reflection spectra of organic compounds. Spectrochimica Acta 17, 698-709 (1961)

Falbe, J., Regitz, M.: RÖMPP Lexikon Chemie, 10. Auflage, p. 933 and 4564. Georg Thieme Verlag, Stuttgart (1999)

Henderson, M.A.: The interaction of water with solid surfaces: fundamental aspects revisited. Surf. Sci. Rep. 46, 1-308 (2002)

Miranda, P.B., Xu, L., Shen, Y.R., Salmeron, M.: Icelike water monolayer adsorbed on mica ar room temperature. Phys. Rev. Lett. 81, 5876-5879 (1998)

Miura, K., Yamada, T., Ishikawa, M., Okita, S.: Apparent contrast of molecularly thin films of water at ionic crystal surfaces. Appl. Surf. Sci. 140, 415-421 (1999)

Rimstidt, J.D.: Quartz solubiltiy at low temperatures. Geochimica et Cosmochimica Acta 61, 2553-2558 (1997)

Sadtchenko, V., Conrad, P., Ewing, E.: $\mathrm{H}_{2} \mathrm{O}$ adsorption on $\mathrm{BaF}_{2}(111)$ at ambient temperatures. J. Chem. Phys. 116, 4293-4301 (2002)

Smith, R.S., Dohnálek, Z., Kimmel, G.A., Teeter, G., Ayotte, P., Daschbach, J.L., Kay, B.D.: In: Buch, V., Devlin, J.P. (eds.) Water in Confining Geometries, pp. 337-357. Springer Verlag, Berlin, Heidelberg, New York (2002)

Thiel, P.A., Madey, T.E.: The interaction of water with solid surfaces: fundamental aspects. Surf. Sci. Rep. 7, 211-385 (1987) 